\title{
A new extension of exponential distribution with statistical properties and applications
}

\author{
Abdullah M. Almarashi ${ }^{a}$, M. Elgarhy ${ }^{b, *}$, Mamhoud M. Elsehetryc $^{c}$, B. M. Golam Kibria ${ }^{d}$, Ali Algarni ${ }^{a}$ \\ a Statistics Department, Faculty of Science, King AbdulAziz University, Jeddah, Kingdom of Saudi Arabia. \\ ${ }^{b}$ Vice Presidency for Graduate Studies and Scientific Research, University of Jeddah, Jeddah, KSA. \\ ${ }^{c}$ Institute of Statistical Studies and Research (ISSR), Department of Mathematical Statistics, Cairo University, Egypt. \\ ${ }^{d}$ Department of Mathematics and Statistics, Florida International University, Miami, FL 33199, USA.
}

\begin{abstract}
A new extension of exponential distribution, named as the Type I half logistic exponential distribution is introduced in this paper. Explicit expressions for the moments, probability weighted, quantile function, mean deviation, order statistics, and Renyi entropy are investigated. Parameter estimates of the new distribution are obtained based on maximum likelihood procedure. Two real data sets are employed to show the usefulness of the new distribution.
\end{abstract}

Keywords: Exponential distribution, maximum likelihood method, moments, order statistics, type I half logistic-G distributions.

2010 MSC: 60E05, 62E10, 62N05.

(C)2019 All rights reserved.

\section{Introduction}

In the last few years, new generated families of continuous distributions have attracted several statisticians to develop new models. These families are obtained by introducing one or more additional shape parameter(s) to the baseline distribution. Some of the genrated families are: the beta-G by Eugene et al. [10], gamma-G (type 1) by Zografos and Balakrishanan [17], Kumaraswamy-G by Cordeiro and de Castro [6], gamma-G (type 2) by Ristic and Balakrishanan [16], transformed-transformer (T-X) by Alzaatreh et al. [2], Weibull-G by Bourguignon et al. [3], exponentiated half-logistic-G by Cordeiro et al. [5], type I half logistic-G family by Cordeiro et al. [4], Garhy-G by Elgarhy et al. [9], exponentiated Weibull-G by Hassan and Elgarhy [12], Kumaraswamy Weibull-G by Hassan and Elgarhy [13], type II half logistic-G by Hassan et al. [14], exponentiated extended-G by Elgarhy et al. [8], Odd Frechet-G by Haq and Elgarhy [11], and

*Corresponding Author

Email addresses: aalmarashi@kau.edu.sa (Abdullah M. Almarashi), m_elgarhy85@yahoo.com (M. Elgarhy),

m_elgarhy85@yahoo.com (Mamhoud M. Elsehetry), kibriag@fiu.edu (B. M. Golam Kibria), ahalgarni@kau.edu.sa (Ali

Algarni)

doi: $10.22436 /$ jnsa.012.03.01

Received: 2018-08-05 Revised: 2018-08-28 Accepted: 2018-09-23 
Muth-G by Almarashi and Elgarhy [1] among others. The cumulative distribution function (cdf) and the probability density function (pdf) of type I half logistic-G family are respectively given by

$$
F(x)=\frac{1-(1-G(x))^{\lambda}}{1+(1-G(x))^{\lambda}}, \quad x, \lambda>0,
$$

and

$$
f(x)=\frac{2 \lambda g(x)(1-G(x))^{\lambda-1}}{\left[1+(1-G(x))^{\lambda}\right]^{2}}
$$

where $\lambda$ is the shape parameter. by

Let, the random variable $\mathrm{X}$ follows the exponential distribution with pdf and cdf are respectively given

$$
\begin{aligned}
& g(x)=\alpha e^{-\alpha x}, \quad \alpha>0, x>0, \\
& G(x)=1-e^{-\alpha x},
\end{aligned}
$$

where, $\alpha$ is the scale parameter.

In this paper, we introduce a new two-parameter model as a competitive extension for exponential distribution using the TIHL-G distributions. The organization of this paper is as follows. In Section 2, we define the type I half-logistic exponential distribution (TIHLE) distribution. In Section 3, we derive a very useful representation for the (TIHLE) density and distribution functions. In the same section, some general mathematical properties of the proposed distribution are given. The maximum likelihood method is applied to drive the estimates of the model parameters in Section 4. A simulation study is carried out to estimate the model parameters of (TIHLE) distribution in section 5. Section 6 gives an illustrative example to explain how the real data sets can be modeled by TIHLE distribution and finally this paper ends with some conclusions in Section 7.

\section{The new model}

In this section, the two-parameter TIHLE distribution is obtained by substituting pdf in (1.3) and cdf in (1.4) into cdf (1.1), then the cdf of type I half-logistic exponential distribution, denoted by TIHLE $(\lambda, \alpha)$ takes the following form

$$
F(x, \varphi)=\frac{1-e^{-\alpha \lambda x}}{1+e^{-\alpha \lambda x}} \lambda, \alpha>0, x>0,
$$

where, $\varphi=(\lambda, \alpha)$ is the set of parameters. Inserting the pdf in (1.3) and cdf in (1.4) into (1.2), we obtain the pdf of TIHLE distribution as the following form

$$
f(x, \varphi)=\frac{2 \lambda \alpha e^{-\alpha \lambda x}}{\left[1+e^{-\alpha \lambda x}\right]^{2}} .
$$

The survival function, hazard rate, reversed-hazard rate and cumulative hazard rate functions of TIHLE distribution are respectively given by

$$
\begin{aligned}
& \overline{\mathrm{F}}(x, \varphi)=1-\mathrm{F}(\chi, \varphi)=\frac{2 e^{-\alpha \lambda x}}{1+e^{-\alpha \lambda x}} \\
& h(x, \varphi)=\frac{f(x, \varphi)}{\overline{\mathrm{F}}(\chi, \varphi)}=\frac{\lambda \alpha}{1+e^{-\alpha \lambda x}}
\end{aligned}
$$




$$
\tau(x, \varphi)=\frac{f(x, \varphi)}{F(x, \varphi)}=\frac{2 \lambda \alpha e^{-\alpha \lambda x}}{1-e^{-2 \alpha \lambda x}}
$$

and

$$
H(x, \varphi)=-\ln (1-F(x, \varphi))=-\ln \left(\frac{2 e^{-\alpha \lambda x}}{1+e^{-\alpha \lambda x}}\right) .
$$

Plots of the pdf and hazard rate function of TIHLE distribution for some values of parameter are displayed in Figures 1 and 2, respectively. It appears from Figure 1 is that the shape of the distribution heavily depends on the value of the parameters. The shape could be uniform to right skewed, which depends on the values of the parameters.

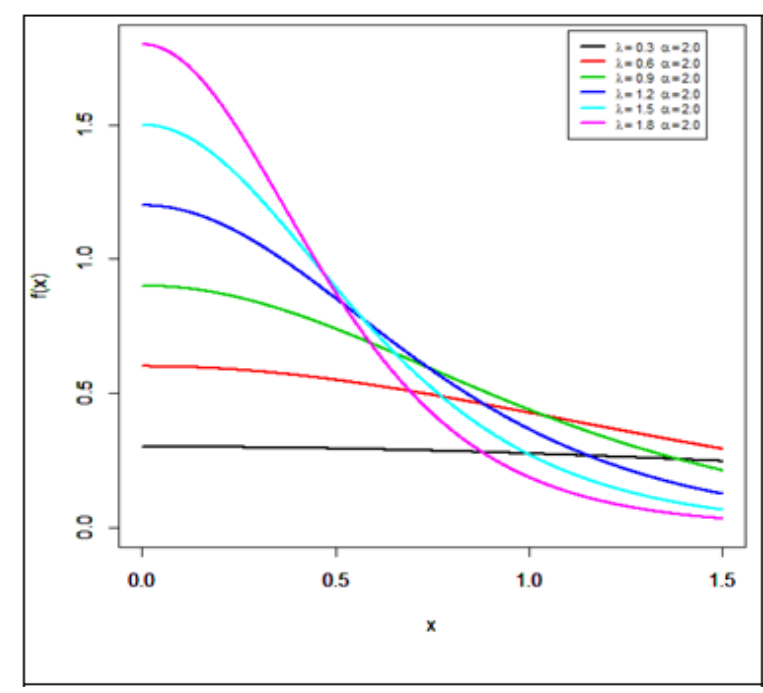

Figure 1: Plots of the pdf of the TIHLE distribution for some Figure 2: Plots of the hazard rate function of the TIHLE distriparameter values.

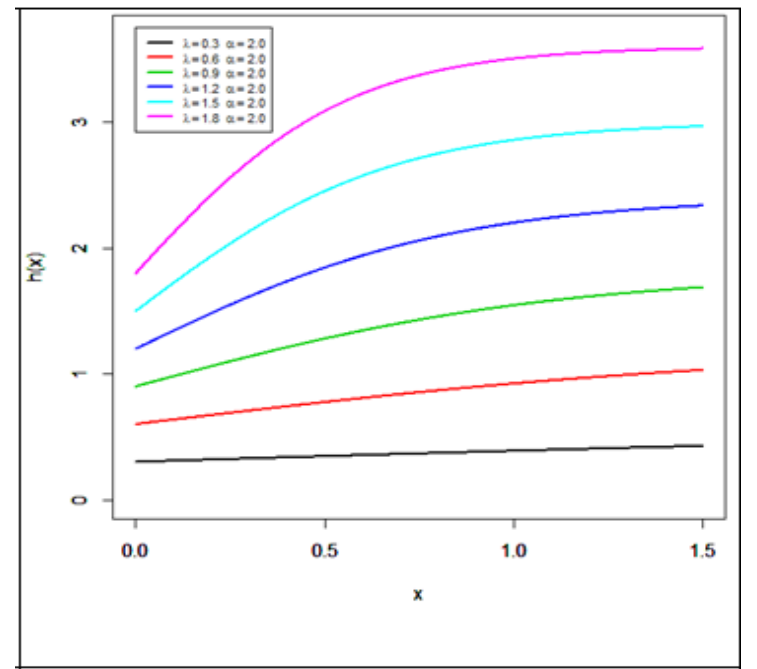
bution for some parameter values.

\section{Statistical properties}

In this section some properties of the TIHLE distribution are obtained.

\subsection{Useful expansions}

In this subsection representations of the pdf and cdf for TIHLE distribution are derived.

Using the generalized binomial theorem, for $\beta>0$ and $|z|<1$,

$$
(1+z)^{-\beta}=\sum_{k=0}^{\infty}(-1)^{k}\left(\begin{array}{c}
\beta+k-1 \\
k
\end{array}\right) z^{k} .
$$

Then, by applying the binomial theorem (3.1) in (2.2), the distribution function of TIHLE distribution becomes

$$
f(x)=\sum_{k=0}^{\infty} \eta_{k} e^{-\alpha \lambda(k+1) x}
$$

where $\eta_{k}=2 \lambda \alpha(-1)^{k}\left(\begin{array}{c}\beta+k-1 \\ k\end{array}\right)$.

An expansion for the cumulative function: 
Using binomial expansion for $[F(x)]^{h}$, where $h$ is an integer, leads to:

$$
[F(x)]^{h}=\left[\frac{1-e^{-\alpha \lambda x}}{1+e^{-\alpha \lambda x}}\right]^{h} .
$$

Using binomial expansion, leads to

$$
[\mathrm{F}(x)]^{\mathrm{h}}=\sum_{z=0}^{\infty} \mathrm{S}_{z} \mathrm{e}^{-\alpha \lambda(z+\mathrm{m}) x^{\beta}},
$$

where $S_{z}=\sum_{i=0}^{h}(-1)^{i+z}\left(\begin{array}{c}h \\ i\end{array}\right)\left(\begin{array}{c}h+z-1 \\ z\end{array}\right)$.

\subsection{Quantile and median}

Quantile functions are used in theoretical aspects of probability theory, statistical applications and simulations. Simulation methods utilize quantile function to produce simulated random variables for classical and new continuous distributions. The quantile function, say $Q(u)=F^{-1}(u)$ of $X$ is given by

$$
u=\frac{1-e^{-\alpha \lambda(Q(u))}}{1+e^{-\alpha \lambda(Q(u))}}
$$

after some simplifications, it reduces to the following form

$$
\mathrm{Q}(\mathrm{u})=\frac{-1}{\alpha \lambda} \ln \left[\frac{1-\mathrm{u}}{1+\mathrm{u}}\right]
$$

where, $u$ is considered as a uniform random variable on the unit interval $(0,1)$.

In particular, the median can be derived from (3.4) by setting $u=0.5$. That is, the median is obtained as

$$
\text { median }=\frac{-1}{\alpha \lambda} \ln \left[\frac{1}{3}\right]
$$

\subsection{Moments}

If $\mathrm{X}$ has the pdf (3.2), then its rth moment can be obtained through the following relation

$$
\grave{\mu}_{\mathrm{r}}=\mathrm{E}\left(\mathrm{X}^{\mathrm{r}}\right)=\int_{0}^{\infty} x^{\mathrm{r}} \mathrm{f}(x) \mathrm{d} x .
$$

Substituting (3.2) into (3.5) yields:

$$
\grave{\mu}_{\mathrm{r}}=\sum_{k=0}^{\infty} \eta_{k} \int_{0}^{\infty} x^{r} e^{-\alpha \lambda(k+1) x} d x .
$$

Then, $\grave{\mu}_{\mathrm{r}}$ becomes

$$
\grave{\mu}_{\mathrm{r}}=\sum_{\mathrm{k}=0}^{\infty} \frac{\eta_{\mathrm{k}} \Gamma(\mathrm{r}+1)}{[\alpha \lambda(\mathrm{k}+1)]^{\mathrm{r}+1}} .
$$

Generally, the moment generating function of TIHLE distribution is obtained through the following relation

$$
M_{X}(t)=\sum_{r=0}^{\infty} \frac{t^{r}}{r !} E\left(X^{r}\right)=\sum_{k, r=0}^{\infty} \frac{t^{r} \eta_{k} \Gamma(r+1)}{r ![\alpha \lambda(k+1)]^{r+1}}
$$


The incomplete moments, say $\varphi_{s}(t)$, is given by

$$
\varphi_{s}(t)=\int_{0}^{t} x^{s} f(x) \quad d x .
$$

Using (3.2), then $\varphi_{s}(t)$ can be written as follows

$$
\varphi_{s}(t)=\sum_{k=0}^{\infty} \eta_{k} \int_{0}^{t} x^{s} e^{-\alpha \lambda(k+1) x} d x
$$

Then, using the lower incomplete gamma function, we obtain

$$
\varphi_{s}(t)=\sum_{k=0}^{\infty} \frac{\eta_{k} \gamma(s+1, \alpha \lambda(k+1) t)}{[\alpha \lambda(k+1)]^{s+1}},
$$

where $v(s, t)=\int_{0}^{t} x^{s-1} e^{-x} d x$ is the lower incomplete gamma function.

Further, the conditional moments, say $\tau_{s}(t)$, is given by

$$
\tau_{s}(t)=\int_{t}^{\infty} x^{s} f(x) d x
$$

Hence, by using the pdf in (3.2), we can write

$$
\tau_{s}(t)=\sum_{k=0}^{\infty} \eta_{k} \int_{t}^{\infty} x^{s} e^{-\alpha \lambda(k+1) x} d x .
$$

Finally, using the upper incomplete gamma function, we obtain

$$
\tau_{s}(t)=\sum_{k=0}^{\infty} \frac{\eta_{k} \Gamma(s+1, \alpha \lambda(k+1) t)}{[\alpha \lambda(k+1)]^{s+1}}
$$

where $\Gamma(s, t)=\int_{0}^{t} x^{s-1} e^{-x} d x$ is the upper incomplete gamma function.

\subsection{Residual life function}

The $\mathrm{n}^{\text {th }}$ moment of the residual life of $\mathrm{X}$ is given by

$$
m_{n}(t)=\frac{1}{R(t)} \int_{t}^{\infty}(x-t)^{n} f(x) d x .
$$

Applying the binomial expansion of $(x-t)^{n}$ into the above formula, we get

$$
m_{n}(t)=\frac{1}{R(t)} \sum_{k=0}^{\infty} \sum_{d=0}^{n}(-t)^{d}\left(\begin{array}{l}
n \\
d
\end{array}\right) \frac{\eta_{k} \Gamma(n-d+1, \alpha \lambda(k+1) t)}{[\alpha \lambda(k+1)]^{n-d+1}},
$$

where $\Gamma(s, t)$ is the upper incomplete gamma function.

The $n^{\text {th }}$ moment of the reversed residual life of $X$ is given by

$$
M_{n}(t)=\frac{1}{R(t)} \int_{0}^{t}(t-x)^{n} f(x) d x .
$$

Applying the binomial expansion of $(t-x)^{n}$ into the above formula, we get

$$
M_{n}(t)=\frac{1}{R(t)} \sum_{k=0}^{\infty} \sum_{d=0}^{n}(-1)^{n+d_{t} d}\left(\begin{array}{l}
n \\
d
\end{array}\right) \frac{\eta_{k} \gamma(n-d+1, \alpha \lambda(k+1) t)}{[\alpha \lambda(k+1)]^{n-d+1}},
$$

where $v(s, t)$ is the lower incomplete gamma function. 


\subsection{Rényi and q-entropies}

The entropy of a random variable $X$ is a measure of variation of uncertainty and has been used in many fields such as physics, engineering and economics among others. The Rényi entropy is defined by

$$
I_{\delta}(X)=\frac{1}{1-\delta} \log \int_{-\infty}^{\infty} f(x)^{\delta} d x, \quad \delta>0 \text { and } \delta \neq 1 .
$$

By applying the binomial theory (3.1) in the pdf (2.2), then the pdf $f(x)^{\delta}$ can be expressed as follows

$$
(f(x))^{\delta}=\sum_{k=0}^{\infty} t_{k} e^{-\alpha \lambda(k+\delta) x},
$$

where $t_{k}=(-1)^{k}(2 \lambda \alpha)^{\delta}\left(\begin{array}{c}2 \delta+k-1 \\ k\end{array}\right)$. Therefore, the Rényi entropy of TIHLE distribution is given by

$$
I_{\delta}(X)=\frac{1}{1-\delta} \log \left[\sum_{k=0}^{\infty} t_{k} \int_{0}^{\infty} e^{-\alpha \lambda[k+\delta] x} d x\right],
$$

That is,

$$
\mathrm{I}_{\delta}(\mathrm{X})=\frac{1}{1-\delta} \log \left[\sum_{k=0}^{\infty} \frac{\mathrm{t}_{\mathrm{k}}}{\alpha \lambda[\mathrm{k}+\delta]}\right]
$$

The q-entropy is defined by

$$
H_{q}(X)=\frac{1}{1-q} \log \left(1-\int_{-\infty}^{\infty} f(x)^{q} d x\right), q>0 \text { and } q \neq 1 .
$$

Therefore, the q-entropy of TIHLE distribution is given by

$$
H_{q}(X)=\frac{1}{1-q} \log \left\{1-\left[\sum_{k=0}^{\infty} \frac{t_{k}}{\alpha \lambda[k+\delta]}\right]\right\} .
$$

\subsection{The probability weighted moments}

The probability weighted moments can be obtained from the following relation

$$
\tau_{r, s}=E\left(X^{r} F(x)^{s}\right)=\int_{-\infty}^{\infty} x^{r} f(x) F(x)^{s} d x
$$

By substituting equations (3.2) and (3.3) into (3.6), replacing $h$ with s, leads to:

$$
\tau_{r, s}=\sum_{k, z=0}^{\infty} \eta_{k} S_{z} \int_{0}^{\infty} x^{r} e^{-\alpha \lambda(k+z+1) x} d x .
$$

Hence, the PWM of TIHLE distribution takes the following form

$$
\tau_{r, s}=\sum_{k, s=0}^{\infty} \frac{\eta_{k} S_{z} \Gamma(r+1)}{[\alpha \lambda(k+z+1)]^{r+1}} .
$$

\subsection{Order statistics}

Let $X_{1: n}<X_{2: n}<\cdots<X_{n: n}$ be the order statistics of a random sample of size $n$ following the TIHLE 
distribution, with parameters $\alpha$ and $\lambda$, then, the pdf of the $k^{\text {th }}$ order statistic ([7]), can be written as follows

$$
f_{k: n}(x)=-\frac{f(x)}{B(k, n-k+1)} \sum_{v=0}^{n-k}(-1)^{v}\left(\begin{array}{c}
n-k \\
v
\end{array}\right) F(x)^{v+k-1},
$$

where, $B(.,$.$) is the beta function. By substituting equations (3.2) and (3.3) in (3.7), replacing h$ with $v+k-1$, leads to

$$
f_{k: n}(x)=-\frac{1}{B(k, n-k+1)} \sum_{\nu=0}^{n-k} \sum_{k, z=0}^{\infty} \eta^{*} e^{-\alpha \lambda[k+z+1] x},
$$

where $\eta^{*}=(-1)^{v}\left(\begin{array}{c}n-k \\ v\end{array}\right) \eta_{k} s_{z}$

Moments of the order statistics is given by:

$$
E\left(X_{k ; n}^{r}\right)=\int_{-\infty}^{\infty} x^{r} f(x) d x
$$

Substituting (3.8) in (3.9) leads to

$$
E\left(X_{k ; n}^{r}\right)=-\frac{1}{B(k, n-k+1)} \sum_{\nu=0}^{n-k} \sum_{k, z=0}^{\infty} \frac{\eta^{*} \Gamma(r+1)}{[\alpha \lambda[k+z+1]]^{r+1}}
$$

\section{Maximum likelihood estimation}

The maximum likelihood estimates (MLEs) of the unknown parameters for the TIHLE distribution are determined based on complete samples. Let $X_{1}, X_{2}, \ldots, X_{n}$ be observed values from the TIHLE distribution with set of parameters $\varphi=(\lambda, \alpha)^{\top}$. The total log-likelihood function for the vector of parameters $\varphi$ can be expressed as

$$
\ln L(\varphi)=n \ln (2 \lambda)+n \ln (\alpha)+n \ln (\beta)-\alpha \lambda \sum_{i=1}^{n} x_{i}-2 \sum_{i=1}^{n} \ln \left(1+e^{-\alpha \lambda x_{i}}\right) .
$$

The elements of the score function $\mathrm{U}(\varphi)=\left(\mathbf{U}_{\boldsymbol{\lambda}}, \mathbf{U}_{\boldsymbol{\alpha}}\right)$ are given by

$$
u_{\lambda}=\frac{n}{\lambda}-\alpha \sum_{i=1}^{n} x_{i}+2 \alpha \sum_{i=1}^{n} \frac{x_{i} e^{-\alpha \lambda x_{i}}}{1+e^{-\alpha \lambda x_{i}}} \quad \text { and } \quad u_{\alpha}=\frac{n}{\alpha}-\lambda \sum_{i=1}^{n} x_{i}+2 \lambda \sum_{i=1}^{n} \frac{x_{i} e^{-\alpha \lambda x_{i}}}{1+e^{-\alpha \lambda x_{i}}} .
$$

Then the maximum likelihood estimates of the parameters $\lambda$ and $\alpha$ are obtained by setting the last two equations to be zero and solving them. Clearly, it is difficult to solve them, therefore applying the NewtonRaphson's iteration method and using the computer package such as Maple or R or other software one can solve these equations and obtain the maximum likelihood estimators.

\section{Simulation study}

It is very difficult to compare the theoretical performances of the different estimators (MLE) for the TIHLE distribution. Therefore, a simulation is needed to compare the performances of the different methods of estimation mainly with respect to their biases, mean square errors and variances (MLEs) for different sample sizes. A numerical study is performed using Mathematica 9 software. Different sample sizes are considered through the experiments at size $n=50,100,150$, and 200. In addition, the different values of parameters $\lambda$ and $\alpha$ are considered.

The experiment was repeated 10000 times. In each experiment, the estimates of the parameters are obtained by maximum likelihood methods of estimation. The means, MSEs and biases for the different estimators are reported from these experiments and presented in Table 1. 
Table 1: The parameter estimation from TIHLE distribution using MLE.

\begin{tabular}{|c|c|c|c|c|c|c|c|c|c|}
\hline $\mathrm{n}$ & Par & Init & MLE & Bais & MSE & Init & MLE & Bais & MSE \\
\hline \multirow{2}{*}{50} & $\lambda$ & 0.9 & 0.9255 & 0.0255 & 0.0271 & 1.5 & 1.5426 & 0.0426 & 0.0739 \\
\cline { 2 - 9 } & $\alpha$ & 0.5 & 0.5142 & 0.0142 & 0.0084 & 0.5 & 0.5142 & 0.0142 & 0.0082 \\
\hline \multirow{2}{*}{100} & $\lambda$ & 0.9 & 0.9136 & 0.0136 & 0.0127 & 1.5 & 1.5191 & 0.0191 & 0.0348 \\
\cline { 2 - 9 } & $\alpha$ & 0.5 & 0.5076 & 0.0076 & 0.0039 & 0.5 & 0.5064 & 0.0064 & 0.0039 \\
\hline \multirow{2}{*}{150} & $\lambda$ & 0.9 & 0.9104 & 0.0104 & 0.0083 & 1.5 & 1.5143 & 0.0143 & 0.0229 \\
\cline { 2 - 9 } & $\alpha$ & 0.5 & 0.5058 & 0.0058 & 0.0026 & 0.5 & 0.5048 & 0.0048 & 0.0025 \\
\hline \multirow{2}{*}{200} & $\lambda$ & 0.9 & 0.9058 & 0.0058 & 0.0059 & 1.5 & 1.5114 & 0.0114 & 0.0172 \\
\cline { 2 - 9 } & $\alpha$ & 0.5 & 0.5032 & 0.0032 & 0.0018 & 0.5 & 0.5038 & 0.0038 & 0.0019 \\
\hline \hline \multirow{2}{*}{50} & $\lambda$ & 2 & 2.0602 & 0.0602 & 0.1355 & 3 & 3.0911 & 0.0911 & 0.3185 \\
\cline { 2 - 9 } & $\alpha$ & 0.5 & 0.5151 & 0.0151 & 0.0085 & 0.5 & 0.5152 & 0.0152 & 0.0088 \\
\hline \multirow{2}{*}{100} & $\lambda$ & 2 & 2.0311 & 0.0311 & 0.0635 & 3 & 3.0513 & 0.0513 & 0.1439 \\
\cline { 2 - 9 } & $\alpha$ & 0.5 & 0.5078 & 0.0078 & 0.0040 & 0.5 & 0.5086 & 0.0086 & 0.0040 \\
\hline \multirow{2}{*}{150} & $\lambda$ & 2 & 2.0186 & 0.0186 & 0.0396 & 3 & 3.0316 & 0.0316 & 0.0908 \\
\cline { 2 - 9 } & $\alpha$ & 0.5 & 0.5047 & 0.0047 & 0.0025 & 0.5 & 0.5053 & 0.0053 & 0.0025 \\
\hline \multirow{2}{*}{200} & $\lambda$ & 2 & 2.0140 & 0.0140 & 0.0292 & 3 & 3.0247 & 0.0247 & 0.0683 \\
\cline { 2 - 9 } & $\alpha$ & 0.5 & 0.5035 & 0.0035 & 0.0018 & 0.5 & 0.5041 & 0.0041 & 0.0019 \\
\hline
\end{tabular}

\section{Data analysis}

In this section, two real data sets are employed to compare the fits of the TIHLE distribution with other exponential (E). For both data, the parameters are estimated by maximum likelihood method. We consider criteria like, Akaike information criterion (AIC), Corrected Akaike information criterion (CAIC), Bayesian information criterion (BIC), Hannan-Quinn information criterion (HQIC), Anderson and Darling test statistic $\left(A^{*}\right)$, and Cramer Von Mises test statistic $\left(W^{*}\right)$. Generally, the lower values of these criteria indicate the better fit to the data.

DATA SET 1: The first data set illustrate the failure and service times for a particular model windshield service times for a particular model windshield of [15]. The data represent the service times of 63 Aircraft Windshield and listed as follows: $0.046,1.436,2.592,0.140,1.492,2.600,0.150,1.580,2.670,0.248,1.719$, $2.717,0.280,1.794,2.819,0.313,1.915,2.820,0.389,1.920,2.878,0.487,1.963,2.950,0.622,1.978,3.003,0.900$, $2.053,3.102,0.952,2.065,3.304,0.996,2.117,3.483,1.003,2.137,3.500,1.010,2.141,3.622,1.085,2.163,3.665$, $1.092,2.183,3.695,1.152,2.240,4.015,1.183,2.341,4.628,1.244,2.435,4.806,1.249,2.464,4.881,1.262,2.543$, 5.140 .

DATA SET 2: The first data set represents 84 observations of failure time for particular windshield model given in Table 16.11 of [15]. The data are recorded as follows: $0.040,1.866,2.385,3.443,0.301,1.876,2.481$, $3.467,0.309,1.899,2.610,3.478,0.557,1.911,2.625,3.578,0.943,1.912,2.632,3.595,1.070,1.914,2.646,3.699$, $1.124,1.981,2.661,3.779,1.248,2.010,2.688,3.924,1.281,2.038,2.82,3,4.035,1.281,2.085,2.890,4.121,1.303$, $2.089,2.902,4.167,1.432,2.097,2.934,4.240,1.480,2.135,2.962,4.255,1.505,2.154,2.964,4.278,1.506,2.190$, $3.000,4.305,1.568,2.194,3.103,4.376,1.615,2.223,3.114,4.449,1.619,2.224,3.117,4.485,1.652,2.229,3.166$, $4.570,1.652,2.300,3.344,4.602,1.757,2.324,3.376,4.663$.

Tables 2 and 4 list the MLEs and their corresponding standard errors (SEs) of the model parameters for data sets 1 and 2, respectively. The numerical values of the AIC, CAIC, BIC, HQIC, $\left(A^{*}\right)$, and $\left(W^{*}\right)$ statistics are listed in Tables 3 and 5 for data sets 1 and 2, respectively. We note that the OGHLE model gives the lowest values for the AIC, CAIC, BIC, HQIC, $\left(A^{*}\right)$, and $\left(W^{*}\right)$ statistics for both data sets among the fitted models. So, the TIHLE distribution performed better than the exponential distribution.

Further, the fitted densities for the first and second data sets are displayed in Figures 3 and 4 (together with the data histogram), respectively. These results illustrate the potentiality of the TIHLE distribution over exponential distribution. 
Table 2: ML estimates of the model parameters and the corresponding SEs for first data set.

\begin{tabular}{|c|c|c|c|c|}
\hline \multirow{2}{*}{ Distribution } & \multicolumn{4}{|c|}{ Estimated parameters and SE } \\
\cline { 2 - 5 } & $\alpha$ & $\lambda$ & SE $(\alpha)$ & SE $(\lambda)$ \\
\hline TIHLE & 6.576 & 0.105 & 4.837 & 0.07686 \\
\hline E & 0.48 & - & 0.06 & - \\
\hline
\end{tabular}

Table 3: Goodness of measures for estimates for first data set.

\begin{tabular}{|c|c|c|c|c|c|c|}
\hline Model & AIC & CAIC & BIC & HQIC & A $^{*}$ & $\mathbf{W}^{*}$ \\
\hline TIHLE & 211.706 & 211.906 & 211.305 & 213.392 & 1.4792 & 0.17189 \\
\hline E & 222.597 & 223.196 & 226.883 & 224.283 & 2.97205 & 0.31826 \\
\hline
\end{tabular}

Table 4: ML estimates of the model parameters and the corresponding SEs for second data set.

\begin{tabular}{|c|c|c|c|c|}
\hline \multirow{2}{*}{ Distribution } & \multicolumn{4}{|c|}{ Estimated parameters and SE } \\
\cline { 2 - 5 } & $\alpha$ & $\lambda$ & $\mathrm{SE}(\alpha)$ & $\mathrm{SE}(\lambda)$ \\
\hline TIHLE & 6.264 & 0.092 & 2.646 & 0.03901 \\
\hline $\mathrm{E}$ & 0.391 & - & 0.043 & - \\
\hline
\end{tabular}

Table 5: Goodness of measures for estimates for second data set.

\begin{tabular}{|c|c|c|c|c|c|c|}
\hline Model & AIC & CAIC & BIC & HQIC & A $^{*}$ & W$^{*}$ \\
\hline TIHLE & 307.473 & 307.621 & 307.321 & 309.427 & 6.2578 & 0.49244 \\
\hline E & 329.754 & 330.603 & 334.616 & 313.708 & 9.63216 & 0.70401 \\
\hline
\end{tabular}

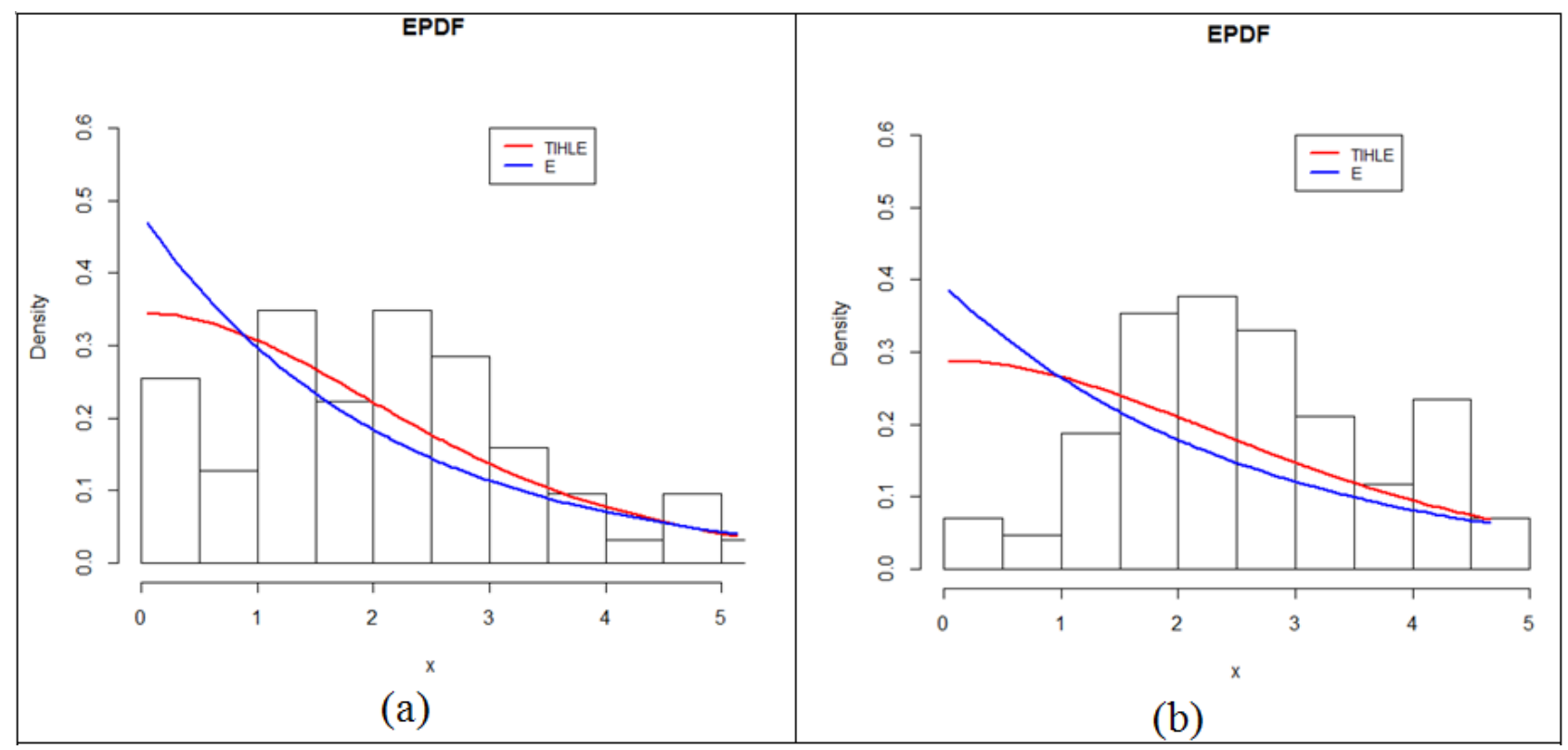

Figure 3: Estimates of the density functions for the: (a) first data and (b) second data. 


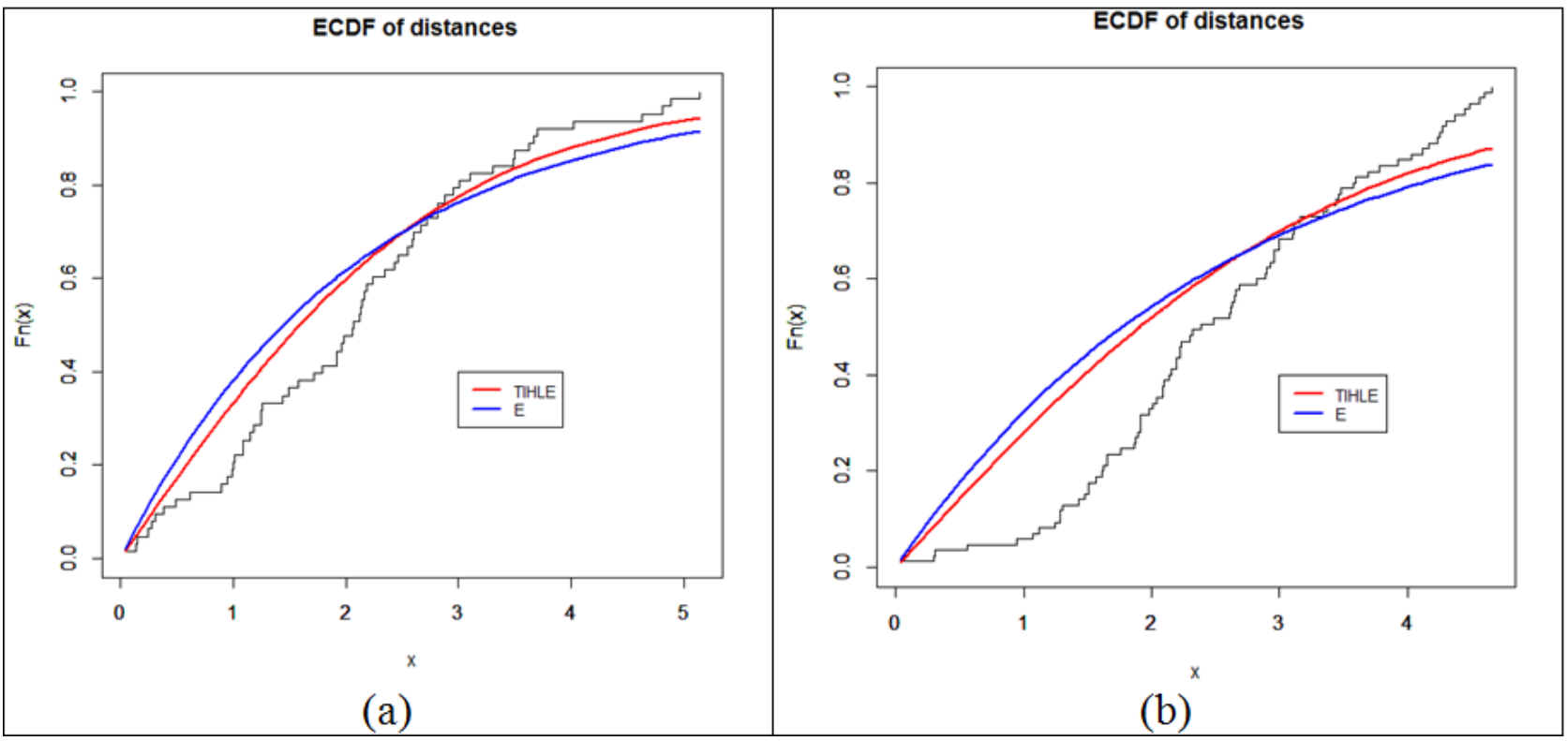

Figure 4: Estimates of the distribution function and empirical distribution (a) first data and (b) second data.

\section{Conclusions}

This paper developed a new extension of the exponential distribution, named as the Type I half logistic exponential distribution. Some of the important properties of the distribution, namely, the moments, probability weighted moments, quantile function, mean deviation, order statistics and Renyi entropy are investigated. Maximum likelihood method is used to estimate the parameters of the new distribution. Two real data sets are employed to show the usefulness of the new distribution. Hope this distribution will be useful for the researchers of various fields.

\section{Acknowledgment}

This article was partially completed while the author, B. M. Golam Kibria was on sabbatical leave (Fall 2017). He is grateful to Florida International University for awarding the sabbatical leave which gave him excellent research facilities.

\section{References}

[1] A. M. Almarashi, M. Elgarhy, A new muth generated family of distributions with applications, J. Nonlinear Sci. Appl., 11 (2018), 1171-1184. 1

[2] A. Alzaatreh, C. Lee, F. Famoye, A new method for generating families of continuous distributions, Metron, 71 (2013), 63-79. 1

[3] M. Bourguignon, R. B. Silva, G. M. Cordeiro, The Weibull-G family of probability distributions, J. Data Sci., 12 (2014), 53-68. 1

[4] G. M. Cordeiro, M. Alizadeh, P. R. D. Marinho, The type I half-logistic family of distributions, J. Stat. Comput. Simul., 86 (2015), 707-728. 1

[5] G. M. Cordeiro, M. Alizadeh, E. M. M. Ortega, The exponentiated half-logistic family of distributions: Properties and applications, J. Probab. Stat., 2014 (2014), 21 pages. 1

[6] G. M. Cordeiro, M. de Castro, A new family of generalized distributions, J. Stat. Comput. Simul., 81 (2011), $883-893$. 1

[7] H. A. David, Order statistics, John Wiley \& Sons, New York, (1981). 3.7

[8] M. Elgarhy, M. Haq, G. Ozel, A new exponentiated extended family of distributions with Applications, Gazi University J. Sci., 30 (2017), 101-115. 1

[9] M. Elgarhy, A. S. Hassan, M. Rashed, Garhy-generated family of distributions with application, Math. Theory Model., 6 (2016), 1-15. 1 
[10] N. Eugene, C. Lee, F. Famoye, The beta-normal distribution and its applications, Commun. Stat. Theory Methods, 31 (2002), 497-512. 1

[11] M. Haq, M. Elgarhy, The odd Frechet-G family of probability distributions, J. Stat. Appl. Prob., 7 (2018), 185-201. 1

[12] A. S. Hassan, M. Elgarhy, A New family of exponentiated Weibull-generated distributions, Int. J. Math. Appl., 4 (2016), 135-148. 1

[13] A. S. Hassan, M. Elgarhy, Kumaraswamy Weibull-generated family of distributions with applications, Adv. Appl. Stat., 48 (2016), 205-239. 1

[14] A. S. Hassan, M. Elgarhy, M. Shakil, Type II half Logistic family of distributions with applications, Pak. J. Stat. Oper. Res., 13 (2017), 245-264. 1

[15] D. Kundu, M. Z. Raqab, Estimation of $\mathrm{R}=\mathrm{P}(\mathrm{Y}<\mathrm{X})$ for three-parameter Weibull distribution, Stat. Prob. Lett., 79 (2009), 1839-1846. 6

[16] M. M. Ristic, N. Balakrishnan, The gamma-exponentiated exponential distribution, J. Stat. Comput. Simul., 82 (2012), 1191-1206. 1

[17] K. Zografos, N. Balakrishnan, On families of beta- and generalized gamma-generated distributions and associated inference, Statistical Methodology, 6 (2009), 344-362. 1 